\title{
Design of Telemetry System for Low Orbit Satellite Based On Short Message Service of BeiDou III Navigation Satellite System
}

\author{
Suquan Ding \\ Beijing Space Quest CO., LTD., Room 207, Building B, No. 28, Xinjiekouwai Street, Xicheng District, \\ Beijing, China \\ ddssqq@sina.com
}

\begin{abstract}
:
The first two BeiDou III satellites were successfully launched on Nov. 5, 2017. Eight BeiDou III satellites have been launched in Xichang, China. The BeiDou III navigation satellite system is plan to provide basic services to countries along the Belt and Road by launching 18 satellites around 2018, and achieve global coverage by 35 satellites around 2020. In addition to navigation services, the BeiDou III system also provide short message service, which can be used for telemetry of low orbit satellites.

The telemetry system of low orbit satellite is designed based on the short message service of BeiDou III system. The onboard telemetry system consists of short message terminals and antennas. The terminal include power module, serial interface, short message processing module, and power amplifier module. The two-layer patches antenna is designed for the onboard telemetry system. Simulation results show that gain of the antenna is above $-3 \mathrm{dBi}$ in the field of 60 degrees. Simulation results also show that time delay of the telemetry data is less than 1 minute. The link budget shows that the link margin is great than $3 \mathrm{~dB}$.
\end{abstract}

Key words: BeiDou III satellites, short message service, telemetry, short message terminal, two-layer patches antenna

\section{Introduction}

After successful contructions of BeiDou I and II navigation satellite system, the goal of China's BeiDou III navigation satellite system is to provide basic services to the countries along the Belt and Road and in neighboring regions by launching 18 satellites around 2018, and to complete the constellation deployment with launching of 35 satellites by 2020 to provide services to global users ${ }^{[1]}$. The BeiDou space constellation shall gradually take a transition from BeiDou II to BeiDou III and provides open services for users worldwide.

The first two BeiDou III satellites were successfully launched on Nov. 5, 2017, while the third and fourth satellites launched on Jan. 12,2018 , the fifth and sixth satellites launched on Feb. 12, the seventh and eighth satellites launched on March 30, in Xichang satellite launch center of China.

The space segment of Beidou III system is a hybrid navigation constellation consisting of 3 Geostationary Earth Orbit (GEO) satellites, 3
Inclined Geosynchronous Satellite Orbit (IGSO) satellites and 24 Medium Earth Orbit (MEO) satellites ${ }^{[2]}$. According to actual situation, spare satellites may be deployed in orbit. The GEO satellites operate in orbit at an altitude of 35,786 kilometers and are located at $80^{\circ} \mathrm{E}$, $110.5^{\circ} \mathrm{E}$, and $140^{\circ} \mathrm{E}$ respectively. The IGSO satellites operate in orbit at an altitude of 35,786 kilometers and an inclination of the orbital planes of 55 degrees with reference to the equatorial plane. The MEO satellites operate in orbit at an altitude of 21,528 kilometers and an inclination of the orbital planes of 55 degrees with reference to the equatorial plane. In comparison with other navigation satellite systems, the BeiDou system operates more satellites in high orbits to offer better anti-shielding capabilities, which is particularly observable in terms of performance in the low-latitude areas. The BeiDou III constellation is shown in Fig. 1 and their subsatellite track are shown in Fig. 2.

The BeiDou system integrates navigation and communication capabilities for the first time, 
and has five major functions: real-time navigation, rapid positioning, precise timing, location reporting and short message communication services. The short message service can send up to 120 Chinese characters which correspond 240 English letters for a single message ${ }^{[3]}$. The short message service provide a way for low cost telemetry system of low orbit satellites. The low cost telemetry system can meet the development of commercial satellites which require to reduce cost greatly.

\section{Design of on board Telemetry system for low orbit satellite based on short message service of BeiDou III sysytem}

Both BeiDou II and BeiDou III system can provide short message services for users. As the Beidou II is a regional navigation system while BeiDou III system aims to provide global services, it is natural to use the BeiDou III system to provide global coverage for the satellite whose telemetry based on short message service.

The system diagram of the onboard telemetry system based on the short message service of BeiDou III system is shown in Fig. 3. The onboard telemetry system consists of short message terminals and antennas. The terminal include power module, serial interface, short message processing module, and power amplifier module. The serial interface receives the telemetry data and connects to the short message processing module which converts the telemetry data based on the short message service protocol. The power amplifier amplifies the output signals of the short message processing module and transmits the signal to the antenna. The power module provides appropriate powers for the serial interface, short message processing module and the power amplifier.

The short message service of BeiDou III system work in $L$ band. The antenna for the onboard telemetry system is selected according to the antenna radiation pattern, polarization characteristics and installation considerations. The two-layer patches antenna is designed for the onboard telemetry system.

\section{Simulation results and link budget}

Simulation results show that gain of the antenna is above $-3 \mathrm{dBi}$ in the field of 60 degrees. The performance of the antenna can meet the requirements of link budget and onboard installation. Simulation results also show that time delay of the telemetry data is less than 1 minute.
The link budgets are computed both for the GEO and MEO satellites. The reqirements for the EIRP of short message terminals for BeiDou system is between $3.5 \mathrm{dBW}$ to 19 $\mathrm{dBW}^{[4]}$ 。 Link budget shows that the link margin is great than $3 \mathrm{~dB}$ when the EIRP is $6 \mathrm{dBW}$ for GEO satellites while the EIRP is $16 \mathrm{dBW}$ for MEO satellites.

\section{Conclusions}

The onboard telemetry system is designed fot low orbit satellites based on the short message services of China's Beidou III navigation satellite system. As the BeiDou III system aims to provide global services around 2020 , it is reasonable to achieve good coverage for telemetry of the user satellites. Simulation results show that the performances of the telemetry system can satisfy the requirements for low orbit satellites. It provides a low cost, space based telemetry solution for commercial low orbit satellites.

\section{References}

[1] China's BeiDou Navigation Satellite System, the State Council Information Office of the People's Republic of China, June 2016

[2] BeiDou Navigation Satellite System Signal In Space Interface Control Document Open Service Signal B3I (Version 1.0), China Satellite Navigation Office, Feb. 2018

[3] Development report on BeiDou navigation satellite system (V2.1), China Satellite Navigation Office, Dec. 2012

[4] General standard of BeiDou navigation satellite system location reporting \& short message communications teminal, China National Administration of GNSS and Applications, Aug. 2014 


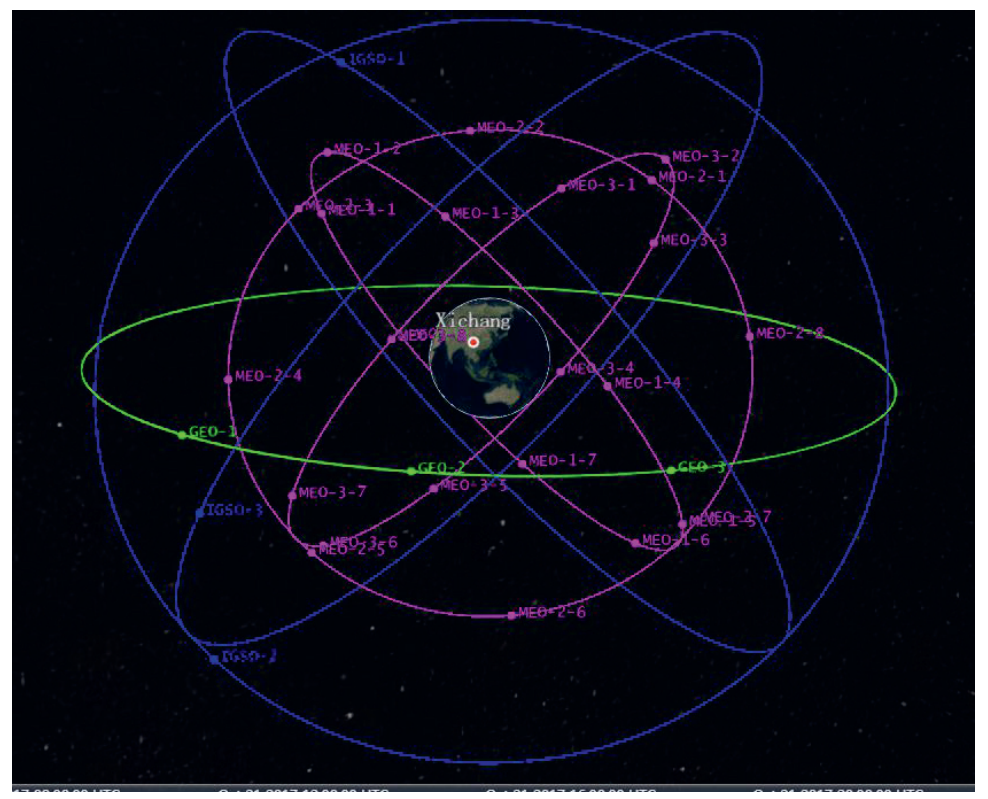

Fig.1. Constellation of BeiDou III navigation satellite system

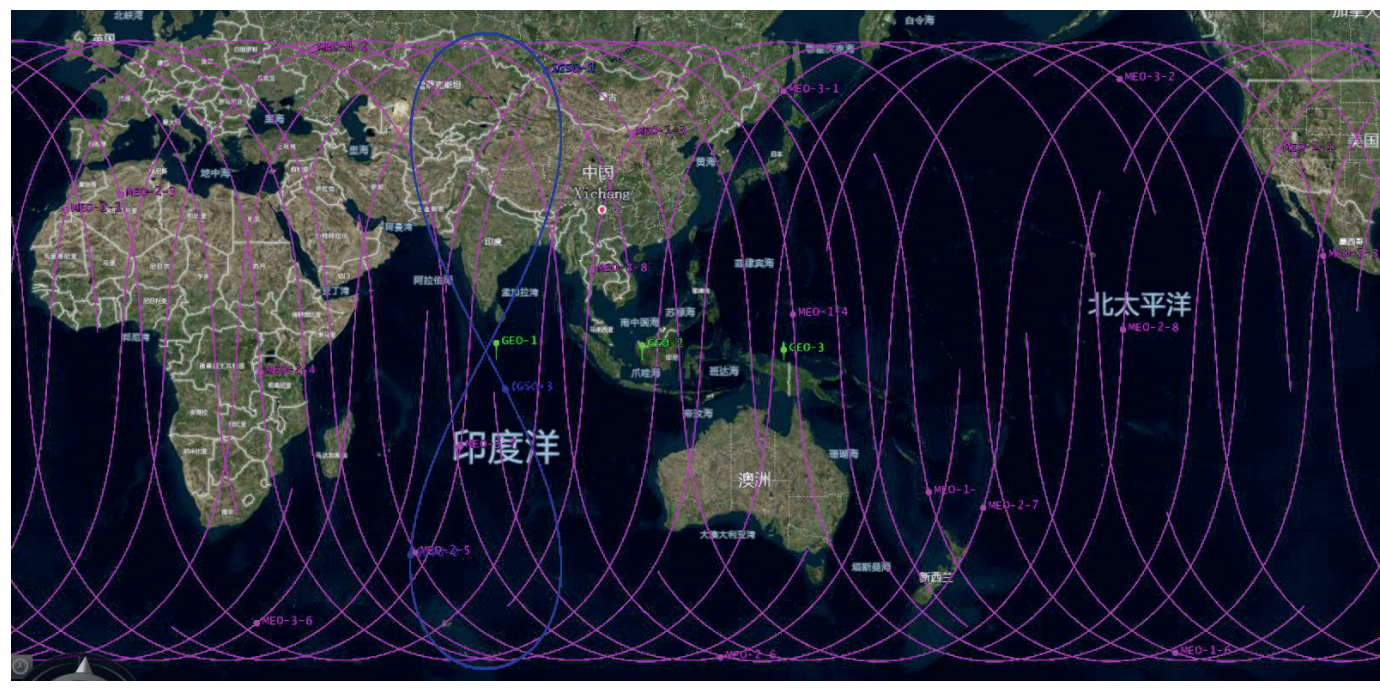

Fig.2.Sub-satellite points of BeiDou III navigation satellite system

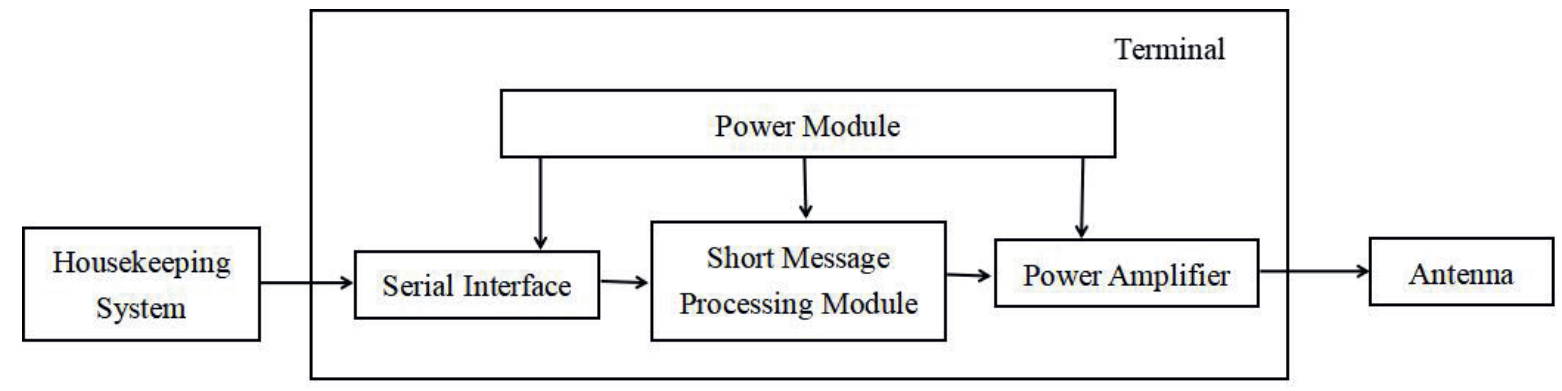

Fig.3.system diagram of the onboard telemetry system 